\title{
Handwriting Skills: Attention Co-varies with Graphic Pattern Stability
}

\author{
P. G. Zanone* J. Boitard ${ }^{*} \quad$ V. Kostrubiec ${ }^{*}$ \\ (*) Université de Toulouse, UPS, PRISSMH, \\ 118 route de Narbonne, F-31062 Toulouse Cedex 9, France \\ E-mail: zanone@cict.fr, kostrubi@cict.fr
}

\begin{abstract}
Attentional cost of graphic patterns generation was investigated using the classic dual-task procedure, in which a concurrent reaction time (RT) task was used as an index of the attentional cost incurred by the motor task. Participants had to trace graphic patterns characterized by $0^{\circ}, 45^{\circ}, 90^{\circ}, 135^{\circ}$ and $180^{\circ}$ relative phase, while responding by a keypress as fast as possible to an auditory signal. The results evidenced a strong and significant correlation between the stability of the produced pattern and the associated attentional cost measures. The size of minor and major axes of the ellipsoids, capturing the amplitude of graphic patterns, decreased as movement frequency increased, as expected by nonlinear models of pattern generation. In cursive writing, this decrease in amplitude with increasing movement frequency is at origin of most frequent letter malformation.
\end{abstract}

\section{Introduction}

A statistical analysis of cursive writing revealed the presence of some particularly troublesome letters, such as $r$ or $k$, which are more often malformed than others, $l, c$ or $u$ [1]. Given that proficiency in handwriting is essential for success at school, it is primordial to understand which cognitive and motor features make some letters more difficult or easier to trace than others.

Our working hypothesis was that troublesome letters involve graphic patterns that are costly attention-wise. To date, attention and handwriting kinematics has been shown to be likened [2-4]. How come then that distinct graphic patterns do require distinct attentional cost in equivalent conditions is still an open question, however. To address this issue, we shall start here from motor control theories providing an account of how graphic patterns are generated and then we infer how this process and attentional cost are related.
Although, in principle, the graphomotor system could generate an infinite number of patterns, in practice it does seldom do so [5]. Among all possible graphic patterns, only few, so-called preferred patterns, are produced with high accuracy and at high frequency [6-7]. The preferred graphic patterns might be conceived of as the basic building blocks for writing, coined as motor primitives or motor prototypes and stored somehow in the motor system [8]. This proposal, however, provides no clue as to how such preferences arise. Under the umbrella of Coordination Dynamics Theory [9] motor preferences depend on the nature of the coupling allowing the graphomotor components to be assembled [9]. If the components were coupled trough linear functions, any motor pattern would be as likely as any another. As they are coupled with nonlinear functions, differences in the stability among patterns arise. Only few patterns are stable enough to be brought about and sustained by the motor system against the perturbations coming from internal and external worlds, thereby defining preferred patterns. Operationally, pattern stability is measured by the variability of produced pattern. The higher the stability is, the less variable the pattern varies across successive productions.

Along this guideline, Athènes and colleagues [1011] investigated the stability of graphic patterns characterized by various values of relative phase. Relative phase amounts to $0^{\circ}$ for right-slanted line, to $180^{\circ}$ for left-slanted line, to $90^{\circ}$ for a circle, to $45^{\circ}$ for right-slated ellipse and to $135^{\circ}$ for left-slanted ellipse. Right-hangers were asked to trace in superposition of each visual model. The graphic performance was assessed trough the produced relative phase. As a result, a classification of graphic patterns was established from the most to the less stable ones $\left(0^{\circ}>\right.$ $45^{\circ}>90-180^{\circ}$ ).

Another line of research on bimanual coordination showed that stability is related to attentional cost [1213]. With the help of the classic dual task procedure, in which a concurrent reaction time (RT) task was used as an index of the attentional cost incurred by the motor

This is an Open Access article distributed under the terms of the Creative Commons Attribution-Noncommercial License 3.0, which permits unrestricted use, distribution, and reproduction in any noncommercial medium, provided the original work is properly cited. 
task, the authors asked participants to produce interlimb $180^{\circ}$ and $0^{\circ}$ patterns while responding as fast as possible to an auditory signal. They demonstrated that $\mathrm{RT}$ is faster for the more stable $0^{\circ}$ than for the less stable $180^{\circ}$.

In the present experiment, we shall test whether attentional cost is related to the stability of relative phase in handwriting. Participants have to produce five graphic patterns: $0^{\circ}, 45^{\circ}, 90^{\circ}, 135^{\circ}$ and $180^{\circ}$ at increasing movement frequency. We hypothesized a linear decrease in the stability accompanied with a linear increase in RT as a function of the required relative phase. The analysis of relative phase, assessing the temporal properties of handwriting generation, was complemented by an analysis of the size of the major and of minor axes of the produced ellipses, capturing the geometrical properties of produced patterns.

\section{Method}

\subsection{Participants}

Eight unpaid, naïve volunteers, 3 males and 5 females, aged about 26 , took part in the study. The study was approved by the local ethical committee of Paul Sabatier University and participants provided a written informed consent in accordance with the Helsinki Declaration.

\subsection{Material and apparatus}

The graphic task was performed on a computercontrolled WACOM (Intuos 2 DTF 720) digitizing tablet, using an electronic pen. As soon as the electronic pen touched the tablet, the $\mathrm{x}$ and $\mathrm{y}$ coordinates of the performed trajectories were digitized at $100 \mathrm{~Hz}$, and stored on a personal computer connected to the tablet for further analysis. The tip of the pen could be localized in both directions $(\mathrm{x}, \mathrm{y})$ with an accuracy of $0.2 \mathrm{~mm}$.

For any given graphic probe, five ellipsoids, characterized by $0^{\circ}, 45^{\circ}, 90^{\circ}, 135^{\circ}$ and $180^{\circ}$ relative phases were generated at random. At any trial, an ellipsoid was displayed in the centre of the white screen of the digitized tablet (see Figure 1). At about $1.2 \mathrm{~cm}$ from the bottom edge of the ellipsoid was displayed a flickering, vertical red stroke, $4 \mathrm{~cm}$ long, specifying the movement frequency. Henceforth, it will be referred to as the visual metronome. The visual metronome specified seven movement frequencies ranging from $1.5 \mathrm{~Hz}$ to $4.5 \mathrm{~Hz}$ by steps of $0.5 \mathrm{~Hz}$. Each blink of the visual metronome lasted 0.05 sec. After each trial, a visual feedback was provided: a Lissajous figure of the produced graphic trajectory was displayed, and lasted on the screen as long as participants desired. The procedure and the visual display were controlled through the Matlab $\mathbb{C}$ Psychophysics Toolbox on a customized $3 \mathrm{GHz}$ PC.

A trigger button was used to record RT response. It was inserted into slant face of a box put on the floor. In each trail, 3 to $142800-\mathrm{Hz}$ auditory stimulus were generated randomly by a microcomputer, with an interstimulus interval ranging from 1 to $5 \mathrm{~s}$. The TR task was controlled by the $\mathrm{C}$ program on a customized $3 \mathrm{GHz} \mathrm{PC}$.

\subsection{Procedure}

Participants were instructed to trace in superposition with the graphic model at the movement frequency prescribed by the visual metronome. Only a counterclockwise rotation of the pen tip was permitted. In RT task, they should respond as soon as possible to auditory signals by pressing the trigger button with their right big toe as soon as possible.

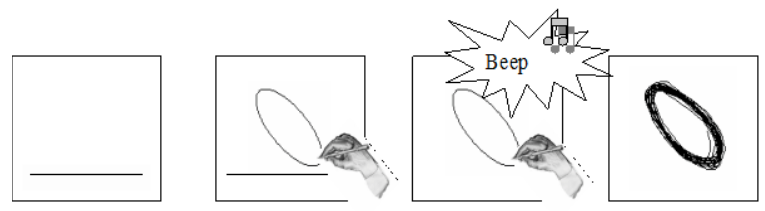

Fig. 1. Graphic trial: visual metronome for 4 sec, followed by the graphic model displayed in the presence of visual metronome for $5 \mathrm{~s}$, followed the graphic model with RT task for 15 s, followed by visual feed-back. Participant traced over the visual model for $20 \mathrm{~s}$.

After familiarization with the graphic and the RT tasks, four blocks of graphic probes were administered. Each bloc involved seven graphic probes, each of them associated to a given movement frequency of visual metronome. The required movement frequencies were presented in increasing order for probe 1 and 3 and in decreasing order for probe 2 and 4. A graphic trial lasted $24 \mathrm{~s}$ and a graphic probe about $13 \mathrm{~min}$. The whole session took about $2 \mathrm{~h}$. To avoid fatigue, a break was proposed after $1 \mathrm{~h}$ of the experimental session.

At the beginning of each graphic trial, a visual prompt reminded the required direction of rotation. During the first four seconds, the visual metronome was turned on, in order to allow participants to capture the required movement frequency (Figure 1). Then, the graphic model was displayed, so that during the next five seconds both the graphic model and the visual metronome were available while participant started tracing the ellipsoid at the required frequency. For the next 20 minutes, the visual metronome was turned off. Participants should maintain the required movement 
frequency while responding as soon as possible to the auditory signal. This procedure avoided subjects being involved in a triple task, that is, to simultaneously follow the metronome, perform the graphic task and respond to auditory signal. A the end of a graphic trial the visual feed-back was displayed, and lasted on the screen as long as participants wished to. Participants were urged to do at their outmost both in the graphic and in the TR task, according to the "optimummaximum" used in the work by the Temprado group.

\subsection{Data Analysis}

For each graphic trial, the number of cycles, the pointestimated relative phase (RP) between $\mathrm{x}$ and $\mathrm{y}$ spatial coordinates, and reaction time (RT) were assessed. The point-estimated RP was defined as the time difference between the consecutive maximal excursions of $\mathrm{x}$ and of $y$ coordinate, reported to the period of the corresponding $\mathrm{x}$ coordinate, and multiplied by $360^{\circ}$. Then, the RP were averaged and corresponding index of variability computed using circular statistics. Note that circular index of variability, $R$, decreases from 1 to 0 as variability increases. For this raison, it will be coined as stability index over the whole article. The accuracy of produced ellipsoid was assessed through constant error (CE), that is, the difference between the produced and required $\mathrm{PR}$, and through absolute error (AE), corresponding to the unsigned value of $\mathrm{CE}$.

For each cycle in a trial, the data were adjusted by the best-fitting ellipse using a least-square procedure. Then, the size of the major and of minor axis of the best fitting ellipse were calculated and averaged. Finally, reaction times, the measure of attentional cost, were defined as the delay between the onset of the acoustic signal and the pressure of the trigger button.

\subsection{Statistical Analysis}

All the data were analyzed using 2 Order (increasing vs. decreasing $) \times 5$ Ellipse $\left(0^{\circ}, 45^{\circ}, 90^{\circ}, 135^{\circ}, 180^{\circ}\right)$ $\times 7$ Frequency $(1.5 \mathrm{~Hz}, 2 \mathrm{~Hz}, 2.5 \mathrm{~Hz}, 3 \mathrm{~Hz}, 3.5 \mathrm{~Hz}, 4$ $\mathrm{Hz}, 4.5 \mathrm{~Hz}$ ) ANOVA. All the variables were treated as repeated measures. If the data did not satisfy the sphericity of variances assumption with regard to Geisser-Greenhouse's index, the required adjustments were applied to the degrees of freedom for the F-tests. The ANOVA model was complemented by linear polynomial contrasts to test whether the distribution of data presents a statistically reliable linear trend.

To assess the correlation between attentional cost and stability, a coefficient of correlation $r$ was calculated between the indexes of stability and corresponding RT for each participant. Then, t-test were applied to the series of coefficients of correlation in order to assess whether their values were significantly higher than -0.5 . For all results, only significant effects at $p<0.05$ are reported.

\section{Results}

\subsection{Number of cycles}

2 (Order) $\times 5$ (Ellipse $) \times 7$ (Frequency) ANOVA on number of cycles revealed a main effect of Frequency $\left(F(1.263,8.841)=59.355, p<0.000, \eta^{2}=0.895\right) . \mathrm{A}$ linear polynomial contrast indicated a significant uptrend $\left(F(1,7)=66.291, p<0.000, \eta^{2}=0.904\right)$, suggesting that in line with task requirements participants increased movement frequency with the visual metronome.

\subsection{Relative phase accuracy}

No significant effects were reported by 2 (Order) $\times 5$ (Ellipse) $\times 7$ (Frequency) ANOVA carried out on CE and $\mathrm{AE}$, ensuring that all the Ellipses were reproduced with a similar accuracy.

\subsection{Stability and attentional cost}

A 2 (Order) $\times 5$ (Ellipse) $\times 7$ (Frequency) ANOVA on the index of stability revealed a main effect of Ellipse $\left(F(1.172,8.207)=13.587, p<0.005, \eta^{2}=\right.$ $0.660)$. A linear polynomial contrast indicated a significant downtrend $\left(F(1,7)=18.392, p<0.004, \eta^{2}\right.$ $=0.724)$, suggesting that stability significantly decreased as a function of the Ellipse. A similar analysis on RT revealed a main effect of Ellipse $\left(F(2.682,18.772)=5.337, p<0.009, \eta^{2}=0.433\right) . \mathrm{A}$ linear polynomial contrast indicated a significant uptrend $\left(F(1,7)=9.295, p<0.019, \eta^{2}=0.570\right)$, suggesting that RT significantly increased as a function of the Ellipse. Mean coefficient of correlation between $\mathrm{R}$ and $\mathrm{RT}$ amounted at $-0.731(\mathrm{SD}=0.293)$. A t-test revealed that the coefficients of correlation were significantly lower than $-0.5(\mathrm{t}(7)=-2.232, p<$ $0.003)$. The indexes of stability and of attentional cost were negatively correlated (Figure 2).

\subsection{The size of major and minor axis of ellipses}

For the major and the minor axis axis, a 2 (Order) $\times 5$ (Ellipse) $\times 7$ (Frequency) ANOVA revealed a main 


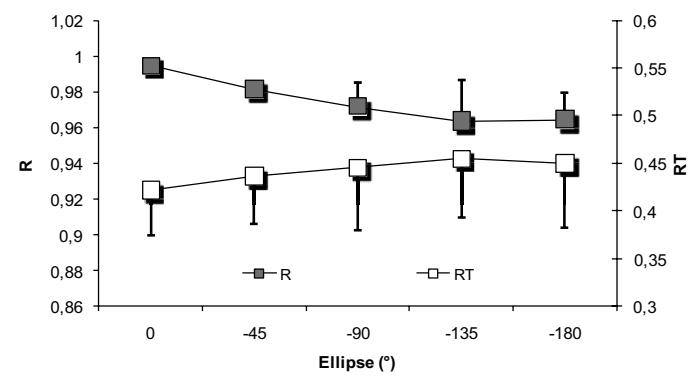

Fig. 2. The index of stability $R$ and of attentionnal cost RT as a function of Ellipse.

effect of Frequency $(F(1.963,13.738)=7.141, p<$ $0.008, \eta^{2}=0.505$, and $F(2.196,15.375)=981.630, p$ $<0.000, \eta^{2}=0.993$, respectively) and of Ellipse $\left(F(1.813,12.689)=64.040, p<0.000, \eta^{2}=0.901\right.$, and $F(1.714,11.997)=21.242, p<0.000, \eta^{2}=0.752$, respectively). Linear polynomial contrasts indicated that both major and minor axes decreased with movement frequency $\left(F(1,7)=29.998, p<0.001, \eta^{2}=\right.$ 0.811 , and $F(1,7)=7.841, p<0.027, \eta^{2}=0.528$, respectively).

\section{Discussion}

In line with our hypothesis, the results indicated that as the required relative phase increased, the TR indicative of the cost incurred by CNS increased and the stability of the produced relative phases decreased. There was also a strong and significant correlation between the stability and attentional cost measures. Such a covariation tends to be a generic property of all coordination systems, at least those involving the oscillation of components of any nature.

Moreover, the amplitude of both axes of the ellipsoids decreased as movement frequency increased, reminding the decreasing in the amplitudes motions expected by classic, nonlinear models [14]. The abovementioned finding might provide some insight into the origins of letter malformation in cursive writing. In the statistical studies, Horton [1] reported that the most frequent malformation pertained to the incorrect size: letters are too small or too large in relation to guide lines: $d$ is written like $a$ or $e$ like $l$.

\section{References}

[1] L. W. Horton, Illegibilities in the cursive handwriting of sixth-graders. The Elementary School Journal, 70(8), 446-450, 1970.
[2] G. Luria, and S. Rosenblum, Comparing the handwriting behaviours of true and false writing with computerized handwriting measures. Applied Cognitive Psychology, 2009; n/a DOI: $10.1002 /$ acp.1621

[3] J.P. Orliaguet, and L.J. Boe, The role of linguistics in the speed of handwriting movements : effects $\mathrm{f}$ spelling uncertainty. Acta Psychologica, 82, 103-113, 1993.

[4] P. Zesiger, P. Mounoud, and C.A. Hauert, Effects of lexicality and trigram frequency on handwriting production in children and adults. Acta Psychologica, 82, 353-365, 1993.

[5] P. Viviani, and R. Schneider, A developmental study of the relationship between geometry and kinematics in drawing movements. Journal of Experimental Psychology : Human Perception and Performance, 17(1), 198-218, 1991.

[6] R. G. J. Meulenbroek, and A. J. W. M. Thomassen, (1991). Stroke-Direction Preferences in Drawing and Handwriting. Human Movement Science, 10(2-3), 247270 .

[7] N. Dounskaia, A.W. Van Gemmert, and G. E. Stelmach, Interjoint coordination during handwriting-like movements. Experimental Brain Research, 135(1), 127$140,2000$.

[8] T. Flash, and B. Hochner, Motor primitives in vertebrates and invertebrates. Current Opinion in Neurobiology, 15, 1-7, 2005.

[9] J.A.S. Kelso, Dynamic Patterns : The self-organization of brain and behavior. Cambridge, Mass : MIT Press, 1995.

[10] S. Athènes, I. Sallagoity, P.G. Zanone, and J.M. Albaret, Evaluating the coordination dynamics of handwriting. Human Movement Science, 23(5), 621-641, 2004.

[11] I. Sallagoïty, S. Athènes, P.G. Zanone, and J. M Albaret, Stability of coordination patterns in handwriting: effects of speed and hand. Motor Control, 8(4), 405-421, 2004.

[12] P.G. Zanone, A. Monno, J.J. Temprado, and M. Laurent, Shared dynamics of attentional cost and pattern stability. Human Movement Science, 20(6), 765-789, 2001.

[13] J.J. Temprado, P.G. Zanone, A. Monno, and M. Laurent, Attentional load associated with performing and stabilizing preferred bimanual patterns. Journal of Experimental Psychology: Human Perception and Performance, 25(6), 1579-1594, 1999.

[14] H. Haken, J.A.S. Kelso, and H. Buntz, A theoretical model of phase. Transitions in human movements. Biological Cybernetics, 51, 347-356, 1985. 